\title{
The Role of Network Architecture in the Onset of Spontaneous Activity
}

\author{
Diletta POZZI ${ }^{a, b^{*}}$, Nicolò MENEGHETTI ${ }^{c}$, Anjan ROY ${ }^{d}$, Beatrice PASTORE ${ }^{a}$, Alberto MAZZONI ${ }^{c}$, \\ Matteo MARSILI ${ }^{\mathrm{d}}$ and Vincent TORRE ${ }^{\mathrm{a}, \mathrm{e}, \mathrm{f}}$ \\ ${ }^{a}$ Neurobiology Sector, International School for Advanced Studies (SISSA), via Bonomea 265, 34136 Trieste, Italy. \\ ${ }^{b}$ Department of Otolaryngology, Head and Neck Surgery, Stanford University School of Medicine, 300 Pasteur Drive, 94305 Stanford, California. \\ Computational Neuroengineering Lab, The Biorobotics Institute, Scuola Superiore Sant'Anna, Viale Rinaldo Piaggio 34, 56125, Pontedera (PI), Italy. \\ ${ }^{d}$ Abdus Salam International Center for Theoretical Physics (ICTP), Strada Costiera 11, 34014 Trieste, Italy. \\ ${ }^{e}$ Cixi Institute of Biomedical Engineering (CNITECH), Ningbo Institute of Materials Technology and Engineering, Chinese Academy of Sciences, Zhejiang, \\ 315201, P. R. China. \\ Center of Systems Medicine, Chinese Academy of Medical Sciences, Suzhou Institute of Systems Medicine (ISM), Suzhou Industrial Park, Suzhou, \\ Jiangsu, 215123 P.R. China.
}

${ }^{*}$ Correspondence: dpozzi@stanford.edu

https://doi.org/10.37175/stemedicine.v1i1.1

\begin{abstract}
Background: The spontaneous activity of neuronal networks has been studied in in vitro models such as brain slices and dissociated cultures. However, a comparison between their dynamical properties in these two types of biological samples is still missing and it would clarify the role of architecture in shaping networks' operation.
\end{abstract}

Methods: We used calcium imaging to identify clusters of neurons co-activated in hippocampal and cortical slices, as well as in dissociated neuronal cultures, from GAD67-GFP mice. We used statistical tests, power law fitting and neural modelling to characterize the spontaneous events observed.

Results: In slices, we observed intermittency between silent periods, the appearance of Confined Optical Transients (COTs) and of Diffused Optical Transients (DOTs). DOTs in the cortex were preferentially triggered by the activity of neurons located in layer III-IV, poorly coincident with GABAergic neurons. DOTs had a duration of $10.2 \pm 0.3$ and $8.2 \pm 0.4$ seconds in cortical and hippocampal slices, respectively, and were blocked by tetrodotoxin, indicating their neuronal origin. The amplitude and duration of DOTs were controlled by NMDA and GABA-A receptors. In dissociated cultures, we observed an increased synchrony in GABAergic neurons and the presence of global synchronous events similar to DOTs, but with a duration shorter than that seen in the native tissues.

Conclusion: We conclude that DOTs are shaped by the network architecture and by the balance between inhibition and excitation, and that they can be reproduced by network models with a minimal number of parameters.

Keywords: Neuronal network · Calcium imaging · GABAergic neuron · Power law · Neural model

\section{Introduction}

A spontaneous electrical activity represents the noise in the nervous system that underlies its operation (1). As opposite, artificial computing elements such as electronic devices have a very low intrinsic noise and are almost noise-free. How the brain copes with its background noise is a major issue of computational neuroscience.

(c) The Author(s). 2019 This is an Open Access article distributed under the terms of the Creative Commons License (http://creativecommons.org/licenses/by/4.0/) which permits unrestricted use, distribution, and reproduction in any medium or format, provided the original work is properly cited.
The spontaneous activity of several brain regions - and in particular of the cortex - shows rapid transitions between periods of intense and synchronous firing (Up states) and of reduced or almost absent electrical activity (Down states) (2-4). These transitions have been observed in rodents performing a variety of tasks $(2,5-7)$, in monkeys (8) and also in vitro in cortical slices (9-11), even when afferent cortical inputs were destroyed. These observations show that an intermittent behavior is intrinsic to the neuronal network and is not caused by the modulation of external inputs (12). These transitions occur at different frequency 
ranges depending on the brain region: in the cerebral cortex, they occur in the range of slow oscillations $(0.5-1 \mathrm{~Hz})(3)$ whereas in the hippocampus mainly in the theta frequency range (4 - $8 \mathrm{~Hz}$ ) (13). Moreover, some slow oscillations are observed in the presence of a coordinated firing between the hippocampus and the cortex (14). In order to explain these transitions, several models of interacting excitatory and inhibitory neurons have been proposed (15) in which intermittency is explained as originating primarily from synaptic noise (16). In alternative models, based on simple sets of differential equations, intermittency has a more deterministic origin $(17,18)$ in a way reminiscent of what observed in chaotic systems. However, it is still to be addressed if this intermittency arises because of the mere biophysical properties of randomly interconnected neurons, or if the network cyto-architecture plays important roles instead. A functional characterization of the same neuronal population preserving the original cytoarchitecture and after dissociation allows evaluating the role of the network architecture in shaping its spontaneous activity.

In this manuscript, we characterized the spontaneous electrical activity of cortical and hippocampal slices from GAD67-GFP mice (19) using conventional calcium imaging $(5,20)$. In this transgenic mouse line, inhibitory GABAergic (GABA+) neurons are labeled with the green fluorescent protein (GFP) so it is possible to identify these neurons and to characterize their role. Here we aim to recover global dynamical properties of cortical and hippocampal networks and a single neuron resolution is not necessary. Therefore, we used calcium imaging with conventional wide-field fluorescence microscopy and not two-photon imaging (21). In our experimental conditions, we obtained an optical trace averaged along the $\mathrm{z}$-axis from which we could identify the onset of Diffuse Optical Transients (DOTs, characterized by a synchronous activation extending in all directions) and Confined Optical Transients (COTs), which were limited to sparse and uncorrelated events.

In addition to the spontaneous activity of slices preserving their original connectivity, we analyzed calcium transients from neuronal cultures obtained after dissociation of the cortex and hippocampus. In these experiments, given the single layer organization of the networks under investigation, calcium transients originating from individual neurons (either GABA+ or GABA-) were unequivocally identified. Modelling with simplified neural networks shows that the dynamical differences of the spontaneous activity between slices and dissociated cultures are due to the multilayer organization of the native tissue.

\section{Materials and Methods \\ Ethical approval}

All procedures were in accordance with the guidelines of the Italian Animal Welfare Act, and their use was approved by the Local Veterinary Service, the SISSA Ethics Committee board and the National Ministry of Health (Permit Number: 2848 - III/15) in accordance with the European Union guidelines for animal care (d.1.116/92; 86/609/C.E.). The animals were anaesthetized with $\mathrm{CO}_{2}$ and sacrificed by decapitation, and all efforts were made to minimize suffering.

\section{Slice preparation}

Slices formed by organotypic cultures were prepared from hippocampal and cortical region of GAD67-GFP mice brain, according to the Gähwiler method (22). Hippocampus together with entorhinal cortex was isolated from 4-6-day-old (P4-P6) postnatal animals. The isolated tissue was cut into $350 \mu \mathrm{m}$ thick transverse slices by means of a tissue chopper (McIlwain, UK). The slices were placed in GBSS solution $\left(\mathrm{g} / \mathrm{l}: \mathrm{CaCl}_{2} \cdot 2 \mathrm{H}_{2} \mathrm{O}\right.$ 0.22; $\mathrm{KCl} 0.37 ; \mathrm{KH}_{2} \mathrm{PO}_{4} 0.03 ; \mathrm{MgCl}_{2} \cdot 6 \mathrm{H}_{2} \mathrm{O} 0.21 ; \mathrm{MgSO}_{4} \cdot 7 \mathrm{H}_{2} \mathrm{O}$ 0.07; $\mathrm{NaCl} 8 ; \mathrm{NaHCO}_{3}$ 0.227; $\mathrm{Na}_{2} \mathrm{HPO}_{4}$ 0.12) added with Kynurenic acid (final concentration $1 \mathrm{mM}$ ) and D-glucose (final concentration $28 \mathrm{mM}$ ), and kept at 4 degrees for 1 hour. Slices were then attached on a glass coverslip $12 \cdot 24 \mathrm{~mm}$ (OrsaTec) with reconstituted chicken plasma (Sigma) coagulated by thrombin (200 U/ml - Merck). After coagulation the coverslips were inserted into plasticone side flat tubes (Nunc) with $0.75 \mathrm{ml}$ of medium containing 25\% horse serum (Gibco), 50\% basal medium Eagle (Gibco) added with L-glutamine, and 25\% Hanks balanced salt solution (Gibco), enriched with glucose to a concentration of $28 \mathrm{mM}$. The tubes were kept in a roller drum rotating ( 10 revolutions per h), in incubator at $37^{\circ} \mathrm{C}$.

In a separate set of experiments, organotypic cultures were prepared according to the Stoppini method (23). The procedure was identical to the Gähwiler method until the cutting and incubation in GBSS solution at 4 degrees. Slices were then placed on sterile, transparent semiporous membranes in six well multiwell (Falcon), with 1 $\mathrm{ml}$ of Neurobasal medium added of B27 supplement (ThermoFisher), the same medium that was used for dissociated cell cultures. The multiwell were kept in an incubator at $37^{\circ} \mathrm{C}$, with $5 \%$ of $\mathrm{CO}_{2}$. In both protocols, the culture medium was changed every five days.

\section{Dissociated cell cultures preparation}

Cortical and hippocampal dissociated cell cultures were prepared from GAD67-GFP mice (P0-P1). Glass coverslips (15 mm diameter) were coated with $50 \mu \mathrm{g} / \mathrm{ml}$ poly-L-ornithine (Sigma-Aldrich, St. Louis, MO, USA) overnight, and just before cells' seeding, a thin layer of Matrigel (diluted 1:50 with culture medium; Corning, Tewksbury MA, USA) was applied. The cortex and hippocampus were isolated and dissected separately. In both cases, the cultures were prepared at two different cell's concentrations. After enzymatic and mechanical dissociation, cells were resuspended at a concentration of $1(2) \cdot 10^{6}$ cells $/ \mathrm{ml}$ in minimum essential medium (MEM) with GlutaMAXTM supplemented with 10\% dialyzed fetal bovine serum (FBS, all from Thermo Fisher Scientific, Waltham, MA, USA), 0.6\% D-glucose, $15 \mathrm{mM}$ Hepes, $0.1 \mathrm{mg} / \mathrm{ml}$ apo-transferrin, $30 \mu \mathrm{g} / \mathrm{ml}$ insulin, $0.1 \mu \mathrm{g} / \mathrm{ml}$ D-biotin, $1 \mu \mathrm{M}$ vitamin B12 and $2.5 \mu \mathrm{g} / \mathrm{ml}$ gentamycin (all from Sigma-Aldrich). Cells 
were then plated at a density of $500(1000)$ cells $/ \mathrm{mm}^{2}$ on glass coverslips. After incubation for $30 \mathrm{~min}$ at $37{ }^{\circ} \mathrm{C}$, the growing medium was added consisting of Neurobasal medium supplemented with B27, GlutaMAXTM and $2.5 \mu \mathrm{g} / \mathrm{ml}$ gentamycin (all from ThermoFisher). Half of the medium was changed after $48 \mathrm{~h}$ adding $2 \mu \mathrm{M}$ cytosine$\beta$-D-arabinofuranoside (Ara-C; Sigma-Aldrich). The neuronal cultures were maintained in an incubator at $37{ }^{\circ} \mathrm{C}, 5 \% \mathrm{CO}_{2}$ and $95 \%$ relative humidity. Half of the medium was again changed once per week.

\section{Calcium imaging}

Calcium imaging experiments on organotypic slices were performed using the Rhod-3 Calcium Imaging Kit (Thermo Fisher) for non-ratiometric analysis. According to the manufacturer's instructions, the samples were incubated for $45^{\prime}$ at room temperature in Ringer solution (145 mM NaCl, $3 \mathrm{mM} \mathrm{KCl,} 1.5 \mathrm{mM} \mathrm{CaCl}_{2}, 1 \mathrm{mM} \mathrm{MgCl}_{2}$, $10 \mathrm{mM}$ glucose and $10 \mathrm{mM}$ Hepes, $\mathrm{pH}$ 7.4) containing the red-fluorescent dye at a concentration of $10 \mu \mathrm{M}$.

Calcium imaging experiments on dissociated cell cultures were performed using the non-ratiometric calcium-binding dye Fura Red, AM (Thermo Fisher), since we observed frequent cells' toxicity when incubating dissociated neurons with Rhod-3. The samples were incubated for $45^{\prime}$ at room temperature in Ringer solution (145 mM NaCl, $3 \mathrm{mM} \mathrm{KCl}, 1.5 \mathrm{mM} \mathrm{CaCl}_{2}, 1 \mathrm{mM} \mathrm{MgCl}_{2}$, $10 \mathrm{mM}$ glucose and $10 \mathrm{mM}$ Hepes, $\mathrm{pH}$ 7.4) containing the red-fluorescent dye at a concentration of $5 \mu \mathrm{M}$. Pluronic F-127 20\% solution in dimethyl-sulfoxide (Thermo Fisher Scientific) was added at a ratio 1:1 with the dye for increasing cells' permeability.

After incubation, both type of samples were transferred to a glass-bottom Petri dish in order to allow visualization in a Nikon Eclipse Ti-U inverted microscope equipped with an HBO $103 \mathrm{~W} / 2$ mercury short arc lamp (Osram, Munich, Germany), a mirror unit (exciter filter BP 465$495 \mathrm{~nm}$, dichroic $505 \mathrm{~nm}$, emission filter BP 515-555) and an Electron Multiplier CCD Camera C9100-13 (Hamamatsu Photonics, Japan). Images were acquired using the NIS Element software (Nikon, Japan) with an S-Fluor 20X/0.75 NA objective and 512.512 spatial resolution. To avoid saturation of the signals, excitation light intensity was attenuated by ND4 and ND8 neutral density filters (Nikon). The recordings were performed at room temperature.

Cortex and hippocampus of a same slice were imaged subsequently. Given the slow time course of measured calcium transients, an acquisition rate of $3-10 \mathrm{~Hz}$ was found to be adequate. In some control experiments, we reached an acquisition rate of $50 \mathrm{~Hz}$ by increasing the intensity of the illuminating light and the binning of pixels; in this case, the total recording time was 5 minutes. During our optical recordings some dye bleaching occurred and the exponential decline of the basal fluorescence was corrected with our custom-made software.

\section{Data Analysis}

\section{Preprocessing of image sequences}

The pre-processing of the acquired videos consisted of two steps. First, we removed the bleaching - driven drift in the z-profile's baseline via linear de-trending. The drift has been corrected by interpolating the points belonging to the baseline in in order to get a function $z_{(t)}$ : then every $\mathrm{i}$-th frame has been multiplied by $\mathrm{z}_{(0)} / \mathrm{z}_{(\mathrm{i})}$. This operation was performed image-wise (i.e., the videos' z-profile has been computed as the mean intensity value of each frame) for control recordings and pixel-wise (i.e., the bleaching has been corrected considering the intensity profile of each pixel separately) for TTX-APV recordings. Second, we measured the changes in fluorescence intensity over time $\left(\Delta \mathrm{F} / \mathrm{F}_{(0)}\right)$ using the open source ImageJ plugin dFoFmovie-CatFullAutoSave.ijm (https://gist.github.com/ ackman678/11155761).

\section{Image sequence analysis}

We identified the activity events as follows. The first step was to binarize each image with a thresholding procedure defining the active pixels in each image. Thresholds were manually set by the experimenter. Then we defined as event a region of contiguous active pixels larger than $16 \mu \mathrm{m}^{2}$.

Two events sharing pixels in consecutives frames are considered to be the same event propagating through time. We define event-splitting when in the $n+1$ frame there are two (or more) "descendants" events whose areas overlap with the area of a single event in the $n$ frame. We define events-merging when, in the when in the $n+1$ frame, there is one event whose area has an overlap with the area of two (or more) "ancestors" events in the $n$ frame.

Once the events were identified, we computed and analyzed two features: the events duration and the maximal area reached by the event during its lifetime. The event duration is the time from the birth and the death of the event, i.e., the first and last frame in which its area was above threshold. In case of splitting, death is defined as the frame in which the last sub-event dies, and in case of merging birth is the frame in which the first of the two originating events was born. The maximal of the area is the maximum value (as number of $\mu \mathrm{m}^{2}$ occupied) reached by an event, considering all ancestor and descendants. We define at this stage "Diffused Optical Transients" the events whose maximal area exceeds half of the area of the slice under investigation, and all the other events as "Confined Optical Transients". Probability distribution of duration and area of Confined Optical Transients are defined with an exponential binning and fit with a power law distribution. We used $n \_$bins $=12$ for the events duration distribution, and $n \_$bins $=42$ for the events area distribution, but the number of bins has a minimal effect on duration power law and no effect on area power law (see

\section{Supplementary Figure 2).}

The TTX-related image sequences have undergone an additional frequency analysis step, in order to test whether their activity time scales slowed down compared to controls. Using a binary grid of Regions Of Interest, the intensity profile of every region across frames was extracted, and we computed the fast Fourier transform of 
each profile. After averaging over the FFT-transforms of the profiles of different ROIs, the result has been filtered with a Moving Average Filter of order $n=5$ using the Matlab built-in function filtfilt.

\section{Computation of correlation coefficient of optical signals}

The correlation coefficient $\rho(\mathrm{t})_{\text {Peak }}$ between peaks of calcium transients was computed as follows. The times, $t_{i}$, at which transient peaks occurred are presented in a conventional raster plot. The raster plot for neuron $i$ and neuron $j\left(\rho\left(\mathrm{t}_{\mathrm{ij}}\right)_{\text {Peak }}\right)$ was computed by dividing the total recording time into intervals of 1 or $10 \mathrm{~s}$. Thus, if $f_{\text {in }}$ and $f_{\text {in }}$ are the number of calcium transients of neuron $i$ and neuron $j$ in the time interval $\Delta t_{n}, \rho\left(\mathrm{t}_{\mathrm{ij}}\right)_{\text {Peak }}$ is computed as follows:

$$
\rho(\mathrm{t} i j) \text { Peak }=\frac{\sum_{n} f_{i n} f_{j n}}{\sqrt{\left(\sum_{n} f_{i n}^{2}\right)\left(\sum_{n} f_{j n}^{2}\right)}}
$$

\section{Statistical analysis}

Data are shown as the mean \pm standard error of the mean from the number of samples indicated in each experiment (see Results and figures captions). Distributions of data from two different data series were compared using the nonparametric test Kruskal-Wallis. The distance between experimental distributions and the corresponding theoretical binomial distributions was calculated using the nonparametric Kolmogorov-Smirnov test. The mean values from two or three data series were compared with One-Way ANOVA and Wilcoxon rank-sum tests. All statistical tests were performed using Matlab software.

\section{Power law fitting}

We followed the methods described in Clauset et al. 2009 (24) to assess the quality of our fit with power law distributions and determine the optimal power law parameters. The maximum likelihood estimator alpha (see Eq. 3.1 in Clauset et al. 2009) and the lower bound of the power law behavior $\mathrm{x}_{\text {min }}$ were estimated simultaneously according to the goodness-of-fit based method. We have a sufficiently large dataset to use the continuous approximation for alpha. For each possible choice of $x_{\min }$, alpha is estimated via the method of maximum likelihood, and we calculated the Kolmogorov-Smirnov goodnessof-fit statistic D (see Eq. 3.9 in Clauset et al 2009). We then select as our estimate of $\mathrm{x}_{\min }$, the value that gives the minimum value of $\mathrm{D}$.

Once we defined the optimal parameters for the power law fit we performed a goodness of fit test of the power law hypothesis (see section 4 of Clauset et al 2009). We generated 1000 power-law distributed synthetic data sets with scaling parameter alpha and lower bound $\mathrm{x}_{\min }$ equal to those of the distribution that best fits the observed data. We have fit each synthetic data set individually to its own power-law model and calculated the KS statistic for each one relative to its own model. The fraction of the time that the resulting statistic is larger than the value for the empirical data defines our p-value. Following the indication of Clauset et al 2009 (section 4.1) we ruled out the power law hypothesis for $p \leq 0.1$, otherwise we concluded that the distribution was compatible with a power law.

\section{Neural modelling}

We have developed highly simplified models of neural networks interacting in a single layer. From these models, we extended the modelling to multilayer networks. One key ingredient of these models is the presence of longtailed distributions - more precisely, distributions with a power law behaviour. The technicalities of these models are described in the next paragraphs.

\section{Single Layer models}

We model the system as a network of $\mathrm{N}$ neurons, where each neuron is connected to $\mathrm{NC}$ randomly chosen neurons (Fig. 7A). At each simulation time step, one unit of charge is added to a randomly selected neuron. A threshold potential $\mathrm{V}_{\text {th }}$ is assigned to each neuron, drawn from a probability distribution $\rho\left(\mathrm{V}_{\mathrm{th}}\right)$. As long as the total charge in the neuron is less than its threshold, the neuron remains silent. When the threshold is reached, the neuron "fires". We have studied two classes of models, characterized by the way the firing neuron perturbs its neighbours. In Model 1, the firing neuron distributes all its charge randomly among its NC neighbours, while in Model 2 the firing neuron gives the same fraction of its charge $\varphi$ to its NC neighbours, and resets its own charge to zero. Upon firing, its threshold is reset by drawing it at random from $\rho\left(\mathrm{V}_{\mathrm{th}}\right)$. Its $\mathrm{NC}$ neighbours list is randomly reset as well. If some of the NC neighbours reach their threshold $\mathrm{V}_{\text {th }}$ from the input of the firing neuron, they fire as well and randomly reset their parameters. This continues till no neuron with charge above $\mathrm{V}_{\text {th }}$ remains. Thus, a single neuron firing may start an avalanche propagating throughout the network. All these relaxations happen instantaneously in the simulation time frame. At the subsequent simulation time step, a new charge is injected to a randomly chosen neuron and the process goes on. In order to have a stationary state, we introduced the parameter $\lambda$, representing dissipation as the probability in which the charge of the firing neuron is removed from the network (instead of being distributed to its neighbours). Since dissipation reduces the amount of excitation, we can consider it as a simplified form of inhibition, caused either by the network inhibition or by an intrinsic adaptation/ inactivation.

We define the size of an avalanche (s) as the number of firing events involved in an avalanche, and the volume of the avalanche (v) as the number of distinct neurons taking part in it. We find that if the threshold is constant or if $\rho\left(V_{t h}\right)$ is Gaussian, then the resulting distribution of size of avalanche follows a power law s ${ }^{-\tau}$ with $\tau<2$. In that case, avalanches of all sizes are possible in the network and the network can always distribute all its charge without requiring a global event involving the entire network. However, if $\rho\left(\mathrm{V}_{\text {th }}\right)$ has a long tailed distribution such as a power law $\left(\rho\left(V_{\text {th }}\right) \sim \mathrm{V}_{\text {th }}{ }^{-\alpha}\right.$ with $\left.\alpha<2\right)$, we again obtain a power law distribution for the size of avalanche, but now 
with $\tau>2$. In this case, an average avalanche size for the network exists and - if the dissipation $\lambda$ is small enough - the network needs a global event to relax. Fig. 7C shows these results for a network with $\mathrm{N}=10,000$ neurons, $\mathrm{NC}=10, \alpha=1.5$ and $\lambda=0.01$ in Model 1. For Model 2, we selected $\varphi=0.1$ and $\lambda=0.01$. Some global events appear in these simulations as spikes at $\mathrm{v}=\mathrm{N}$. The global events occurring in these models correspond to the experimentally observed diffuse optical transients, here referred as DOTs (see Results, Fig. 1-4).

For both models, we have also studied variations where $\mathrm{NC}$ is power law distributed, or the number of neurons perturbed at each time step is random. These variations did not change the results as far as the power law and the resonance is concerned. However, as expected, adding charge to multiple neurons at each time step increases the frequency of the avalanches.

\section{Multilayer models}

In order to explore the reason for the broader width of the DOTs in the cortex, we extend the above model to a multilayer model. In the multilayer model, each layer has a similar structure as in the previous model, but now every neuron in each layer also has one connection to a neuron in the next layer (Fig. 7E). Following the same rules as for the single layer case, a neuron transfers its charge randomly among its $\mathrm{NC}$ neighbours in the same layer and one neighbour in the next layer. After the stability of all neurons in a layer is confirmed like in the single layer case, we check for unstable neurons in the next layer and continue this process. Note that all this sequence happens at the same simulation time step, before some charge is added to another random neuron. However, we assume that the relaxation in subsequent layers happen with a time delay $\Delta \tau$ and we used it while plotting the time series. In Fig. 8F and 8I we show that indeed a broad DOT, reminiscent of the structure observed in experiments, can appear in such a simple cyclic multi-layer model. This happens because of multiple simultaneous DOTs appearing in the different layers but with a time delay $\Delta \tau$. These DOTs appear in a cyclic fashion; a DOT appearing in any of the layer triggers a DOT in the next layer and it continues through the consecutive layers till all the unstable neurons relax, either by transferring their charge or by dissipating them. The quantity $\Delta \tau$ is ad-hoc in the current analysis and indicates the existence of functional layers interacting with each other with a time delay. In simulations, its exact value can be adjusted, along with the number of neurons perturbed at each time step, in order to obtain a time series reminiscent of the one observed in experiments.

\section{Results}

The mouse cortex is composed of 6 layers (LI-VI) and is approximately $500-1000 \mu \mathrm{m}$ thick (Figure 1A) depending on the region (25). The most superficial layer (LI) is $50-80 \mu \mathrm{m}$ thick and is primarily composed of $\mathrm{GABA}+$ neurons, while excitatory neurons are located in layers II-VI (see for a review (26)). The hippocampal system consists of the dentate gyrus (DG), the four regions of the cornu ammonis (CA1-3) fields and the subiculum (Sub) (Supplementary Figure 1A). Our imaging experiments were performed with a $20 \mathrm{X}$ objective in areas corresponding to cortical layers I-IV and hippocampal CA1-3, all of them expressing GABA+ neurons.

The slices were prepared from the medial temporal lobe overlying the hippocampus, corresponding to the entorhinal cortex. The native connectivity between the two regions was maintained. In a separate set of experiments instead, the cortex and hippocampus from the same slice were mechanically separated. After 1 or 2 weeks in culture, a thickness of $50-70 \mu \mathrm{m}$ was measured (see Supplementary Figure 2) corresponding to approximately 5 - 6 cellular layers. When imaging the slices with a conventional epi-fluorescence microscope, the identification of calcium transients at the single cell level was distorted - at some extent - by the light scattering from nearby focal planes. Nonetheless, we were able to identify the emergent collective properties of the cortical and hippocampal networks.

\section{The spontaneous activity in cortical networks}

Slices were stained with the fluorescent Calcium indicator Rhod 3 - AM (Figure 1B). The spontaneous activity was recorded by measuring optical transients using a high resolution and high sensitivity EM-CCD camera (Supplementary Video 1) acquiring images at $5-50 \mathrm{~Hz}$. The total recording time for each session varied from 10 up to 40 minutes and, during this time, the emitted fluorescence $\mathrm{F}_{(\mathrm{t})}$ could decrease by 10-30\%, because of dye bleaching. Following correction for the bleaching, the fractional optical signal $\Delta \mathrm{F} / \mathrm{F}_{(0)}$ was computed (Supplementary Video 2), assigning $\mathrm{t}=0$ at the beginning of the recording, from the entire imaged slice (blue trace in Figure 1D) and therefore referred as $\Delta \mathrm{F} / \mathrm{F}_{\text {network }}$. In addition, $\Delta \mathrm{F} / \mathrm{F}_{(0)}$ was computed from regions of interest (ROIs) corresponding to active cortical areas comprising both GABA+ and GABA- neurons (colored traces in Figure 1D). We observed confined optical transients (COTs) in areas ranging from few tens (corresponding to 1-5 cells) up to $11.000 \mu^{2}$ (see areas inside red circles in Figure 1E). Moreover, we observed diffused optical transients (DOTs) which invaded almost all layers of the visualized slice (Figure 1F) with a frequency varying from 0.2 to 4 per minutes. After computing the $\Delta \mathrm{F} / \mathrm{F}_{\text {network}}$, DOTs appear as large peaks with a fast rising time, which was in the order or below our recording time resolution (20 - $200 \mathrm{~ms})$.

The distribution of the maximal area of observed optical transients (COTs and DOTs) was broad and exhibited a power law behavior over almost 3 log units (Figure 1G), with an average slope of $1.87 \pm 0.42$ (see Table 1). The duration of COTs did not follow a power law instead (Figure 1H). In 22 out of 27 slices analyzed, the distribution of maximal areas had an outlier point corresponding to the occurrence of DOTs (see the arrows in Figure 1G). The number of distinct COTs observed in the same frame fluctuated in time around a mean value of 30 (Figure 1I). During the appearance of a DOT, the 


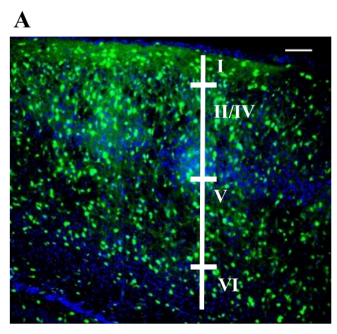

D
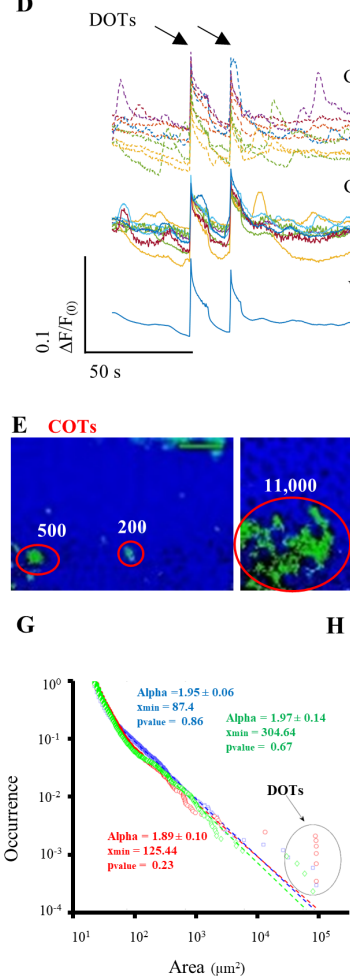
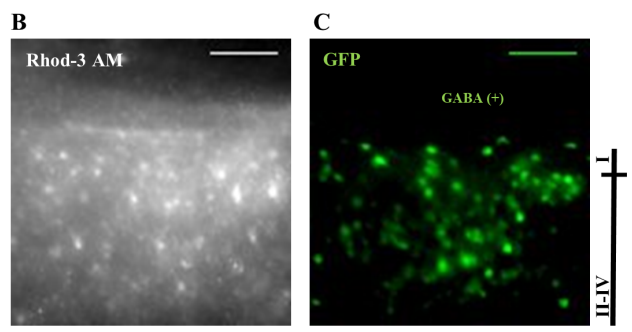

DOTs

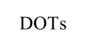

$>\lambda$

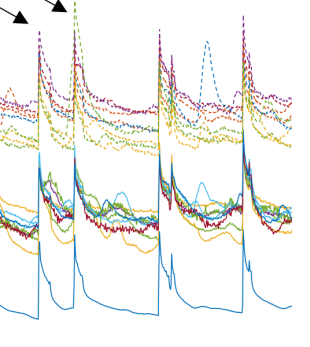

F DOTs
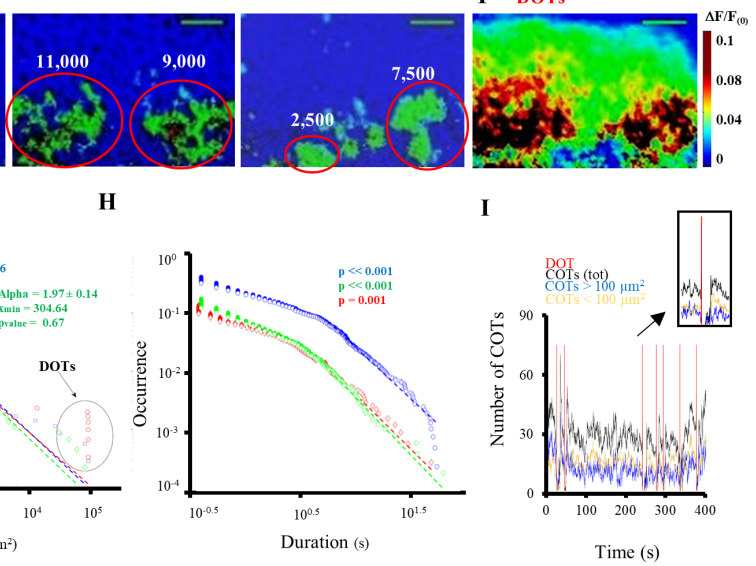

Figure 1: Spontaneous calcium transients in the cortex. (A) Fluorescent image of cerebral cortex from a GAD67-GFP mouse (P5) with DAPI nuclear staining; GABA+ neurons are labeled in green. The white vertical bar indicates the putative layers. (B) Organotypic slice from the same mouse line loaded with the fluorescent indicator Rhod-3 AM during calcium imaging, and corresponding GABA+ neurons (C) in the same field of view. The black vertical bar on the right indicates the approximate limits of cortical layers in this example. (D) $\Delta F / F_{(0)}$ traces from the whole image (blue trace at the bottom, defined as $\Delta F / F_{\text {network }}$ ) and from selected $G A B A+($ colored traces in the middle) and GABA- neurons (colored traces on top) from a calcium imaging recording. (E) Examples of Confined Optical Transients (COTs) from the pseudo-color $\Delta F / F_{(0)}$ movie of the recording, showing isolated regions of variable sizes (see red circles; the rounded numbers are expressed in $\mu \mathrm{m}^{2}$ ). $(\mathrm{F})$ Example of Diffuse Optical Transient (DOT), where the increase in $\Delta F / F_{\text {network }}$ involves more than $50 \%$ of the imaged slice. Color bar on the right. Scale bars: $80 \mu \mathrm{m}$ in both fluorescence and pseudo-color images. (G, H) Probability distribution of area and duration of COTs in the cortex. Circles, rhombus and asterisks represent data from three representative experiments: the corresponding dotted lines in $(\mathrm{H})$ show the linear fitting for each distribution. DOTs are visible as resonances outside the area's distributions (see black circles). Insets indicates test of power law hypothesis and, only if $p>0.1$, power point slope (alpha) and onset. (I) Density of COTs over time for different area sizes (COTs $<100 \mu m^{2}$ and COTs $>100 \mu m^{2}$, yellow and blue line respectively) and for all sizes (black line) with reference to DOTs occurrence (red vertical lines). The inset shows the decrease in COTs density corresponding to DOTs' occurrence.

number of COTs drastically decreased and increased again at the DOT termination, when the diffuse activation invading almost the entire slice - broke in several smaller active regions (Figure 1I, inset). The intermittency between COTs and DOTs, as well as the power law distributions of maximal area and duration, were similar in hippocampal slices (see Supplementary Figure S1).

\section{Origin of COTs and DOTs}

Recorded optical transients (both COTs and DOTs) could originate from neurons and/or glial cells. In order to evaluate the contribution of glial cells, we compared optical transients before and after the addition of $1 \mu \mathrm{M}$ tetrodotoxin (TTX), a well-known blocker of neuronal activity $(27,28)$. The application of TTX completely abolished DOTs in cortical slices (Figure 2A), but residual optical transients could be still detected. However, these transients had a slower rising phase and time course, as demonstrated by the computation of the power spectrum density PSD: in the presence of TTX, the PSD decreased in an almost continuous trend, while in control conditions the signals exhibited an increase between 0.015 and $0.03 \mathrm{~Hz}$ (Figure 2B). Moreover, when the distribution of maximal areas of events in TTX was subtracted to 
that observed in control (Con) conditions, the resulting distribution was very similar to that seen prior to subtraction, as well as the number of events detected (Figure 2C). For these reasons, we concluded that the large portion of calcium transients observed in control conditions originates primarily from firing neurons and only at a minor extend from glial cells. We cannot exclude, however, the contamination from slow calcium waves driven by metabotropic glutamate receptors present both in neurons and glial cells (29).

After application of the GABA-A receptors antagonist bicuculline $50 \mu \mathrm{M}$ in cortical slices, the size and duration of DOTs visibly increased (Figure 2D). As opposite, the amplitude and duration of DOTs were significantly reduced by the application of $50 \mu \mathrm{M}$ APV (Figure 2E, F), suggesting that DOTs originate primarily from the activation of NMDA receptors $(30,31)$. The distribution of maximal areas of events (Figure 2G) were similar in control conditions and in the presence of APV. Therefore, the activation of NMDA receptors is not the main mechanism causing COTs and that the activation of kainate and AMPA glutamate receptors, together with persistent sodium currents plays an important role (32 - 34). All these experimental observations show that DOTs exhibit a pharmacology very similar to that of usual Up states $(10,35)$.

Optical recordings of DOTs have a sharp rising phase, reminiscent of the rising phase of an action potential in a single neuron. An action potential in a neuron occurs when the summation of synaptic inputs reaches a threshold and a regenerative mechanism is activated. It is conceivable, therefore, that DOTs occur when the overall neuronal activity reaches a given threshold and a regenerative mechanism - operating at the network level causes a collective excitation to occur (36). There is also a possible alternative possibility: the appearance of DOTs is favored or triggered when specific clusters or sets of neurons are activated. In order to test this possibility, we investigated whether DOTs are initiated by the activation of privileged/specific regions - which we refer as starters - or instead their appearance depends on the activation of enough neurons randomly distributed in the slice. In the first case, a statistical analysis could reveal the existence of "starters", i.e. of cortical regions whose activation is more likely to trigger or precede a DOT, in the latter case the appearance of a DOT is caused by the concomitant occurrence of a sufficient number of random events. In order to obtain an adequate statistical verification, we prolonged our imaging experiments to 30 - 40 minutes and occasionally up to 1 hour, so to observe several tens of DOTs. In our long recordings, some bleaching occurred which was compensated and the stained slice exhibited optical transients with the same frequency and amplitude during the total observation time (Figure 3A). All the frames before the occurrence of the DOT were analyzed, and each pixel was binarized with a threshold corresponding to the $30 \%$ of the maximal $\Delta \mathrm{F} / \mathrm{F}_{0}$ from the whole image, so to obtain a map of the seeds of the DOTs (Figure 3B). If these seeds do not have any significant spatial correlation, then the origin of DOTs is random, but if these seeds exhibit some correlation than the existence of starters of DOTs finds some support.

We calculated the spatial overlap between these seeds and we colored the pixels according to their frequency of appearance in the seeds, with red and dark red indicating a high appearance (Figure 3C). If a pixel participated to a seed in a random way, its statistics is expected to follow a binomial distribution $\mathrm{P}(\pi, x, N)(37)$ : in this case if $\pi$ is probability of pixel to be active, the probability $\mathrm{P}$ of $x$ occurrence in series of $\mathrm{N}$ trials is:

$$
P(x)=\frac{N !}{x !(N-x) !} \pi^{x}(1-\pi)^{N-x}
$$

If the frequency of occurrence of pixels in the seeds follows the binomial distribution, than DOTs have a random origin, but if a given pixel occurs in the seeds more often than expected from the binomial distribution, then we can consider it as a starter. We found that red pixels in Figure 3C occurred in seeds more often than expected by the binomial distribution in 6 out of 7 experiments (Figure 3D, E; p $<0.001$ two-sample Kolmogorov-Smirnof test) and therefore are candidates to be starters of the DOTs. In order to identify the neuronal types composing these starter regions, we localized the GABAergic neurons in slices after binarizing the images in the green channel. After comparison with the location of starters in the same slice, we observed a minimal spatial overlap (Figure 3F), suggesting that starters might be composed by clusters of non-GABAergic, probably excitatory neurons. Moreover, a preferential localization of starters at $200-300 \mu \mathrm{m}$ in depth from the outer most layer was observed, which corresponds approximately to layer III (Figure 3G).

\section{COTs and DOTs in hippocampal networks: origin and comparison with the cortex}

We analyzed the probability of existence of DOTs starters in hippocampal slices. In contrast with what seen in cortical slices, the frequency of pixel activation was similar to a theoretical Binomial Distribution in 3 out of 5 experiments (Supplementary Figure 3, p > 0.05 twosample Kolmogorov-Smirnof test). The existence of

Table 1: Power law slopes of area distributions in the cortex and hippocampus.

\begin{tabular}{|l|l|l|}
\hline & Connected Area & Detached Area \\
\hline Cortex & $2.24 \pm 0.27$ & $2.41 \pm 0.56$ \\
\hline Hippocampus & $2.28 \pm 0.26$ & $2.47 \pm 0.34$ \\
\hline
\end{tabular}

Values are expressed as absolute values, mean \pm standard deviation. $n=16 / 8$ cortical/hippocampal recordings in connected tissues; $n=6 / 6$ cortical/hippocampal recordings in detached condition. The binning used for these calculations was 42 . 
starters in hippocampal slices is therefore less probable than in cortical ones. While the same clusters of neurons seem to be involved in the generation of a DOT in the cortex, in hippocampal networks DOTs are more likely to originate when a sufficient number of neurons - randomly distributed - are activated.

Comparing the distributions of area and duration of COTs in the cortex and hippocampus (Figure 4A, B) and the area distribution power law's slopes (Figure 4C), we did not observe significant differences. In addition, very similar distributions and power law slopes of optical signals were obtained after mechanical truncation of the connections between hippocampus and cortex (see Table 1). We next analyzed the duration and frequency of DOTs separately, in 7 representative brain slices that showed a sufficient number of DOTs, and that we were able to record in the cortical and the hippocampal region for the same time (i.e. $10 \mathrm{~min}$ ) without incurring into dye - and phototoxicity-driven tissue damage. The distribution of the duration of DOTs shows that they last longer in the cortex (Figure 4D: $10.21 \pm 0.32 \mathrm{~s}$ for cortex, $8.19 \pm 0.43 \mathrm{~s}$ for hippocampus). On the other hand, the distribution of the Inter Event Intervals (IEIs) shows that hippocampal
DOTs are more frequent (Figure 4E: $67.72 \pm 4.88 \mathrm{~s}$ for cortex, $48.77 \pm 6.23 \mathrm{~s}$ for hippocampus). These differences were statistically significant. Both distributions were fitted with appropriate exponential equations (see blue and red lines in Figure 4D, E).

\section{Dissociated neuronal cultures}

In order to understand if the native cyto - architecture of networks plays a role in determining the frequency, duration and synchronization of spontaneous events, we analyzed the spontaneous activity in dissociated cultures form the cortex and hippocampus of GAD67-GFP mice and compared it to the one observed in slices from the same tissues.

In dissociated cultures, formed by a single layer of cells, neuronal calcium transients have a typical sharp rising phase which is distinguished from those originating from glia cells (38). Dissociated cell cultures are known to exhibit a degree of synchronization depending on the density of the culture (39). In our experiments, we considered two culture densities: $500 / 800 \mathrm{cells} / \mathrm{mm}^{2}$ (medium density, MD; Figure 5A, B, E) and 1000 cells $/ \mathrm{mm}^{2}$

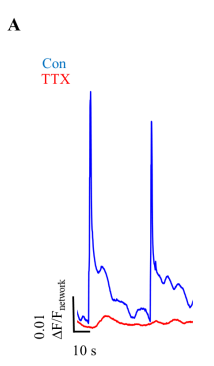

D
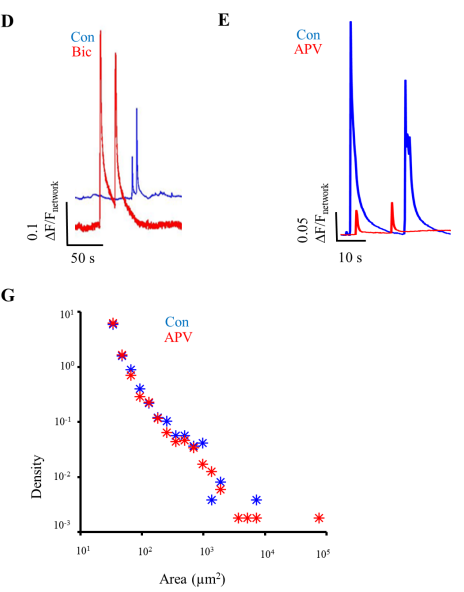

C

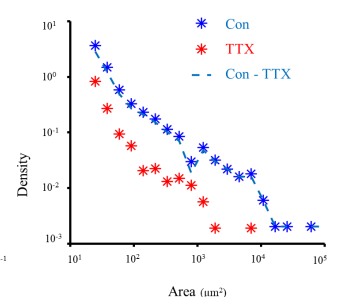

$\mathbf{F}$

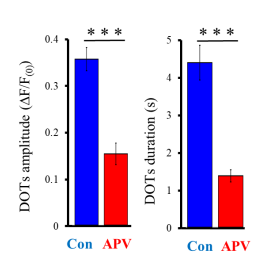

Figure 2: Blockade of voltage - gated sodium channels (by TTX) and glutamate NMDA receptors (by APV) on cortical slices. (A) DOTs observed in control conditions (blue trace) are abolished in the presence of $10 \mu \mathrm{M} T \mathrm{TX}$ (red trace), as shown by the computation of $\Delta \mathrm{F} / \mathrm{F}_{\text {network. }}$ (B) Power Spectrum Density (PSD) computed as the average PSD of optical recordings from small regions corresponding to GABA+ neurons in control (blue traces) and in TTX (red traces) from two representative experiments (rhombus, 41 GABA+ neurons; crosses, 37 GABA+ neurons). (C) Density vs area of events in control (blue marks), in TTX (red marks) and in control-TTX (blue dotted line) in one experiment. From three representative experiments: $\mathrm{n}$ $=16714$ events detected in Con, $n=14007$ in Con-TTX; $n=68948$ in Con, $n=53566$ in Con-TTX; $n=32312$ in Con, $n=21586$ in Con-TTX ( $p>0.05$ in all three cases, Kruskal-Wallis test). (D) $\Delta F / F_{\text {network }}$ in control conditions (blue trace) and after application of Bicuculline $50 \mu M\left(\right.$ red trace). (E) $\Delta F / F_{\text {network }}$ in control conditions (blue trace) and after application of APV $50 \mu \mathrm{M}$ (red trace). (F) Average amplitudes and duration of DOTs in control (blue bars) and in APV (red bars): $0.34 \pm 0.03 \Delta F / F_{(0)}$ and $4.33 \pm 0.49$ s for control, $0.15 \pm 0.02 \Delta F / F(0)$ and $1.32 \pm 0.16$ s for APV. These average values were obtained from 3 experiments in control and APV conditions, subsequently: $n=32$ (3) DOTs (slices) for control, $n=45$ (3) DOTs (slices) for APV. ${ }^{~} p<0.001$ Kruskal Wallis test. (G) Density vs area of events in control (blue marks) and in APV (red marks) in a representative experiment. 
or more (high density, HD; Figure 5C, D, F). Figure 5 shows the results obtained from cortical cultures of GAD67-GFP mice, in which we could distinguish between GABAand GABA+ neurons by comparing GFP-positive cells (Figure 5B, D) to those stained with the calcium sensitive dye only (Figure $\mathbf{5 A}, \mathbf{C}$ ), and separating the corresponding optical traces (Figure 5E, F). GABA- and GABA+ neurons are representative, with a good approximation, of the excitatory and the inhibitory population respectively.

We found different degrees of synchrony among the peaks of calcium transients in MD and HD cultures. We computed the mean cross-correlation among pairs of neurons $\rho(t)_{\text {Peak }}$ in a time window of $1 \mathrm{sec}$ and we found that $\rho(t)_{\text {Peak }}$ was higher in HD than in MD cultures $(0.604 \pm 0.002$ vs $0.337 \pm 0.002$, Figure 5G). However, in both cases the number of simultaneously active neurons over time showed periods of almost complete synchrony interspersed with epochs of very low coherent electrical activity (Figure 5H). The probability distribution of active neurons showed a power law behavior with slope-2 when the number of neurons were less than some tens: this distribution exhibited also outliers corresponding to the total number of neurons recorded, very similar to DOTs in slices (Figure 5I). We obtained similar results from dissociated hippocampal cultures (see Supplementary Figure 4). The average duration and IEI of these highly synchronous events - merging data from MD and HD cultures - were $3.31 \pm 0.18 \mathrm{~s}$ and $24.99 \pm 1.24 \mathrm{~s}$, significantly decreased comparing to the DOTs observed in slices (Figure 5L, M). For this comparison, we used slices cultured in the same medium (Neurobasal/B27) used for dissociated cells, in order to avoid changes in neuronal synchronization due to the effect of different chemicals in the growing media (40).

These results suggest that DOTs are present both in native and in dissociated networks, and synchrony arises independently from the network architecture.

We aimed to investigate more in detail the spontaneous activity arising from GABAergic, inhibitory neurons. We found that an unequivocal distinction between GABA+ and GABA- neurons was possible only in dissociated MD cultures, in which neurons are not totally confluent. Thus, we compared calcium transients between GABA+ and GABA- neurons plated at a medium density and we found that the value of $\rho(t)_{\text {Peak }}$ - in a time window of $1 \mathrm{~s}$ - was higher in GABA+ than in GABA- neurons (Figure 6A). We asked whether large calcium transients were more correlated than smaller transients (41) and indeed calcium transients larger than $30 \%$ of the maximal amplitude observed in each trace were more correlated than the smaller ones (Figure 6B). After blockage of GABA-A receptors with $1 \mu \mathrm{M}$ bicuculline, the amplitude of calcium
A

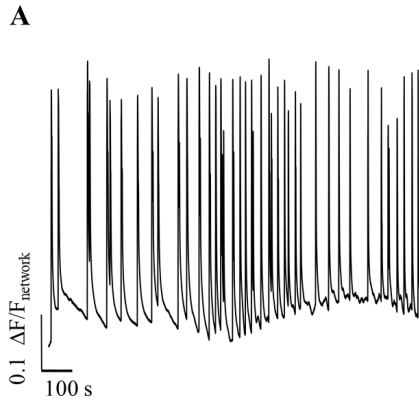

D

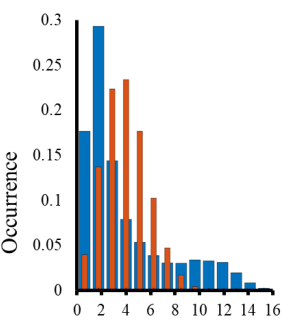

$\mathrm{n}$

$\mathbf{E}$
B
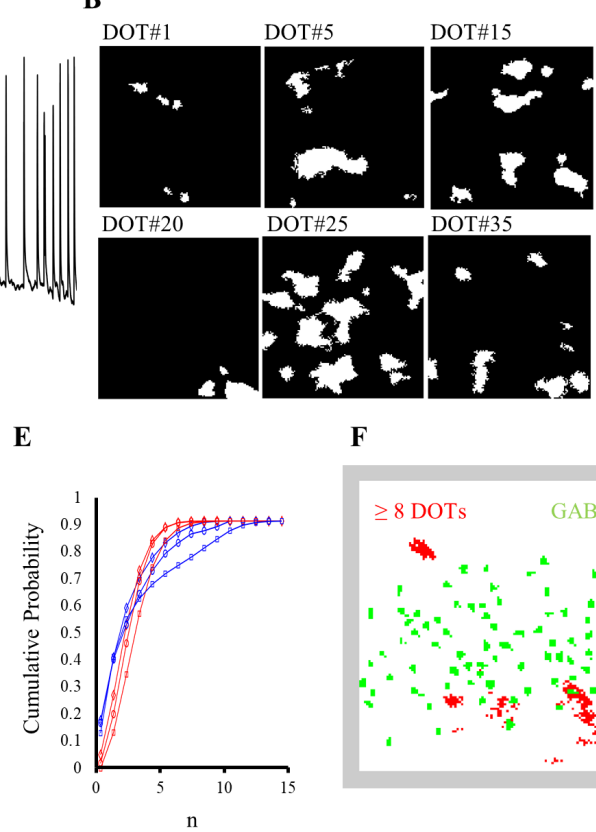

C

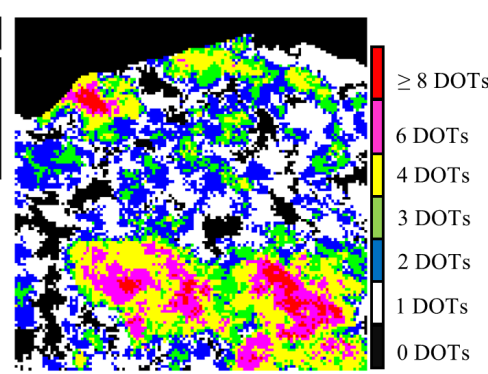

G
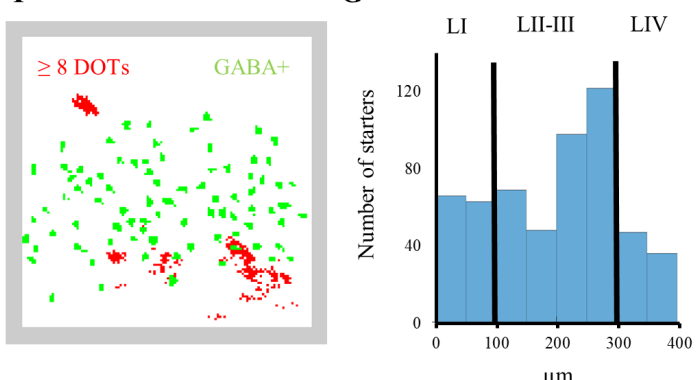

Figure 3: Regions initiating DOTs in cortical slices. (A) $\Delta F / F_{\text {network }}$ of a representative 20 min recording. (B) Binary images of some representative seeds regions identified $200 \mathrm{~ms}$ before 6 randomly selected DOTs (out of a total of 35). (C) Sum of all binary images corresponding to seeds; different colors indicate regions activated before multiple DOTs (see color bar on the right: red regions are activated before the onset of 8-11 DOTs). (D) Probability of activation of a pixel from experimental data (red bars) compared to a binomial distribution (blue bars) in the experiment shown in A - C. The X-axis represents the number of trials (frames) considered. (E) Cumulative probability of pixel activation from experimental data (blue lines) compared to the corresponding binomial distributions (red lines) in three representative experiments. The two distributions were significantly different $(p<0.001$ two-sample Kolmogorov-Smirnof test). (F) Regions activated before the occurrence of 8 - 11 DOTs (red pixels) in the example shown in A - C, with reference to the location of GABA+ neurons (green pixels). (G) Distribution of starters location among cortical layers. $n=7$ slices. The density is higher between LII and LIII. 
transients became uniform in both populations (Figure 6C), however both the rising and falling phase of these transients were slower. Therefore we compared $\rho(t)_{\text {Peak }}$ in a time window of $10 \mathrm{~s}$, which was consistently higher in $\mathrm{GABA}+$ neurons even after the application of bicuculline (Figure 6D). The average IEI between peaks was significantly lower in the GABA+ population (Figure 6E), whereas the amplitude of $\Delta \mathrm{F} / \mathrm{F}_{(0)}$ was similar in $\mathrm{GABA}+$ and GABA- neurons (Figure 6F). In order to identify possible delays in the transmission of excitatory and inhibitory signals, we repeated our imagingexperiments using an acquisition rate of $50 \mathrm{~Hz}$ but we did not detect any consistent delay between GABA+ and GABAneurons at the available temporal resolution of $20 \mathrm{msec}$. Our results suggest that inhibitory GABA+ neurons have more synchronized and frequent spontaneous activity, in agreement with some previous in vivo observations (42).

\section{Simplified models of neuronal networks}

In order to understand the underlying mechanisms of the emergent dynamical properties, we explored highly simplified neural networks (Figure 7A), which have just 3-4 free parameters. This approach is complementary to those developed in current large projects such as the Blue Brain Project, Human Brain Project and Allen Brain Initiative where modeling involves an extremely large number of parameters, in the order $103-104$ times more than in our simplified models. Our models are based on two major assumptions: firstly, dissociated neuronal cultures have a single layer organization, while cortical and hippocampal slices are multilayers; secondly some key factors, such as the threshold $\mathrm{V}_{\text {th }}$ and the number of connections per neurons $\mathrm{NC}$, have a longtailed distribution with a power law behavior. Figure 7B illustrates two representative models, while a detailed description is provided in the Methods section. The charges injected in the network at each time step mimic
A

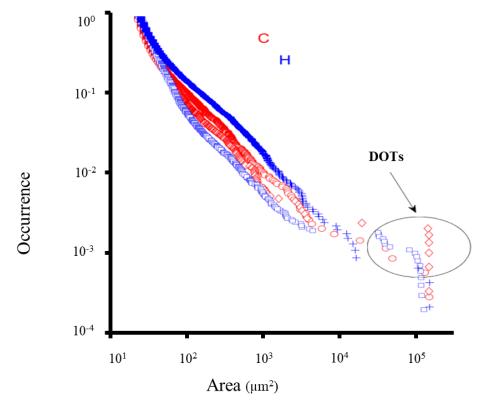

C

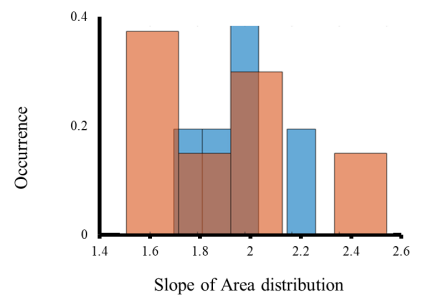

D

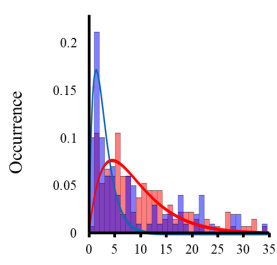

Duration (s)
B
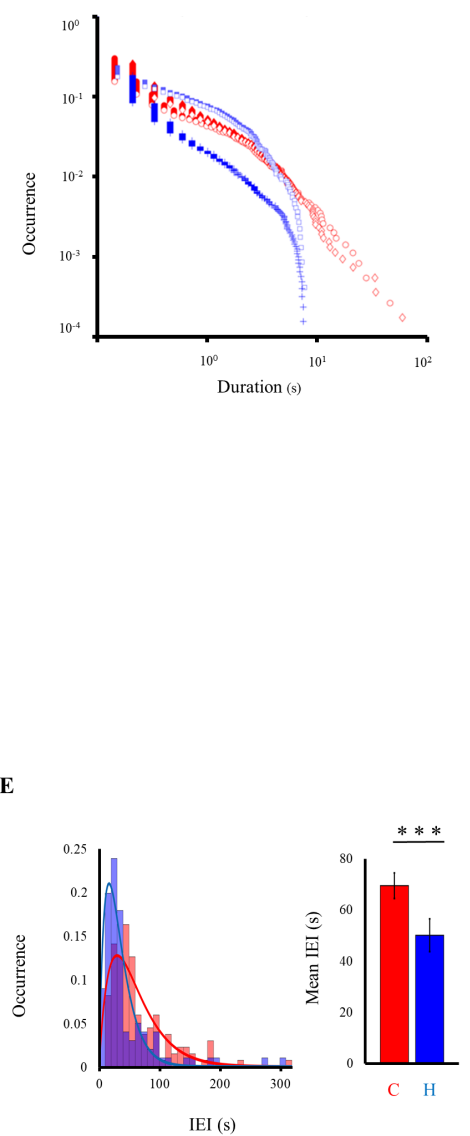

Fig. 4. Dynamics of cortical and hippocampal events compared. (A, B) Power law distributions of area and duration of COTs from four representative experiments with cortical events $(C)$ marked in red, and hippocampal events $(\mathrm{H})$ marked in blue. DOTs are represented as outliers outside the area's distributions (see black circle in A). The area distributions are power laws (power law hypothesis test $p>0.1$ ) and display a slope close to 2. (C) Distribution of power law slopes for area in the cortex (red bars) and hippocampus (blue bars); $n=16$ cortical recordings, $n=8$ hippocampal recordings. (D) Distribution (left panel) and average values (right panel) of cortical (red bars) and hippocampal (blue bars) DOTs durations. The corresponding exponential fitting is $f(x)=0.05^{*} x^{*} \exp (-x / 4.54)$ for the cortex, $f(x)=0.33^{*} x^{*} \exp (-x / 1.39)$ for the hippocampus. (E) Distribution (left panel) and average values (right panel) of Inter Event Intervals (IEIs) in cortical (red bars) and hippocampal (blue bars) slices. The corresponding exponential fitting is $f(x)=0.011^{*} x^{*} \exp (-x / 33)$ for the cortex, $f(x)=0.034^{*} x^{*} \exp (-x / 17)+0.58$ for the hippocampus. $n=102 / 7$ cortical DOTs/slices, $n=137 / 7$ hippocampal DOTs/slices analyzed. ${ }^{* *} p<0.01,{ }^{* *}{ }^{*} p<0.001$ Kruskal-Wallis test. 


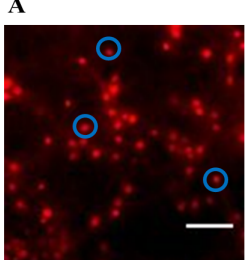

B

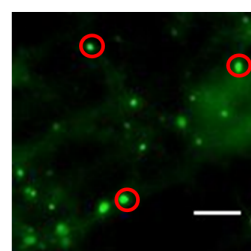

$\mathbf{E}$
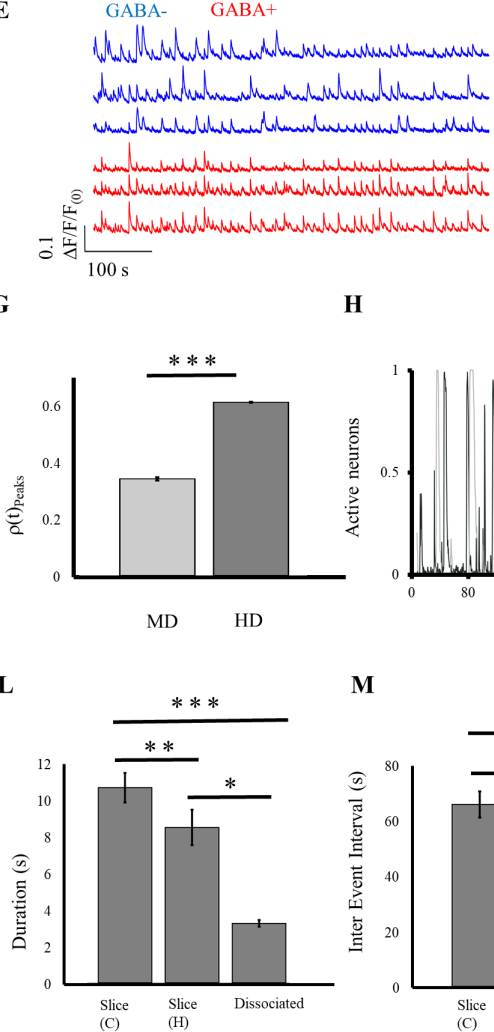

M
C

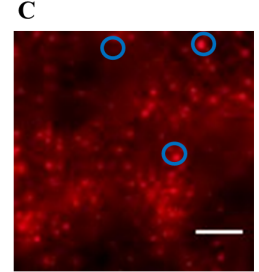

D

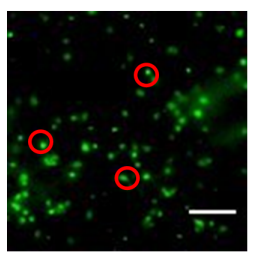

$\mathbf{F}$

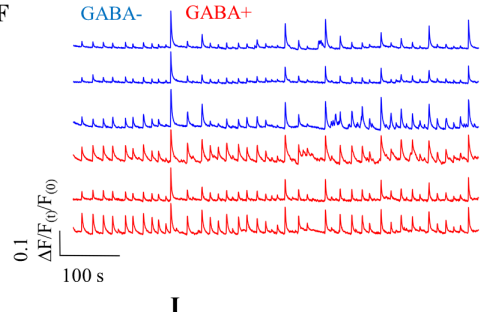

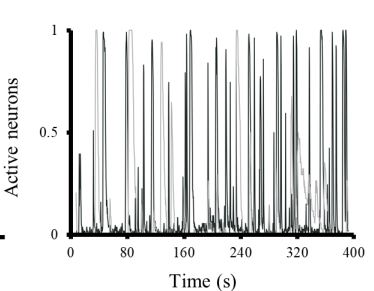
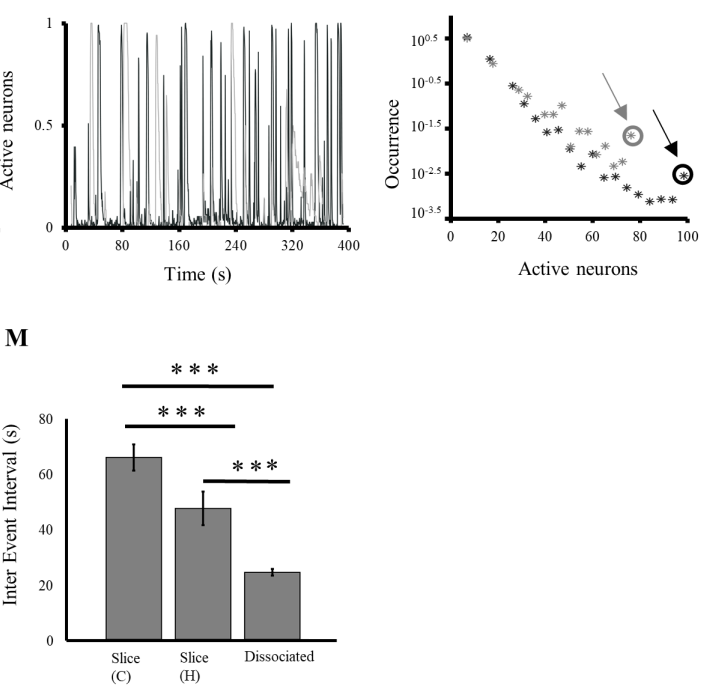

Fig. 5. Dissociated cortical cultures plated at high (HD) and medium (MD) density. (A, C) MD and HD cultures stained with Fura-Red calcium-sensitive dye. (B, D) Corresponding GABA+ neurons in the same field of view. From the neurons in the blue and red circles, the corresponding optical traces $\triangle F / F_{(0)}$ are shown in $(E, F)$ : blue and red traces represent $G A B A-$ and $G A B A+$ neurons respectively. Scale bars, $80 \mu \mathrm{m}$, in all fluorescence images. (G) Average $\rho(\mathrm{t})_{\text {Peak }}$ in MD (light gray) and HD (dark gray) cultures; $n=9354(3)$ pairs of neurons(sample) for HD, $n=1211(3)$ pairs of neurons(sample) for MD. (H) Activation of neurons over time in MD (gray) and HD (black) cultures, normalized to the total number of neurons (105 and 34 for HD and MD cultures, in this representative experiment). (I) Probability distribution of the number of neurons simultaneously active in MD (gray marks) and HD (black marks) cultures, with corresponding outliers (see circles). (L, M) Average duration and IEI (s) of DOTs in cortical (C) and hippocampal (H) slices (same data as in G, $\mathrm{H}, \mathrm{I}$ ) vs dissociated cultures. $\mathrm{n}=78(4)$ DOTs(samples) for dissociated cultures. ${ }^{*} \mathrm{p}<0.51$, ${ }^{*}{ }^{*} \mathrm{p}<0.001$ two-sided Wilcoxon rank-sum test.

random synaptic inputs received by the neurons. Model 1 and Model 2 of a single layer network have just 3 and 4 free parameters, respectively: the threshold $V_{\text {th }}$, the degree of connectivity $\mathrm{NC}$, the strength of connectivity $\varphi$ (only in Model 2) and the rate of dissipation $\lambda$. The dissipation $\lambda$ represents all the biophysical processes reducing the excitation, such as the inhibitory inputs and all mechanisms associated to adaptation and desensitization. We found that when $\mathrm{V}_{\text {th }}$ is a random variable with a long tailed distribution, such as a power law of $\rho\left(\mathrm{V}_{\mathrm{th}}\right)=\mathrm{V}_{\mathrm{th}}^{-\alpha}$ $(\alpha<2)$, both Model 1 and Model 2 reproduce several of our experimental data. In fact, as experimentally observed, they reproduce a power law distribution of the number of simultaneously active neurons with a slope of about -2
(Figure 7C). These models reproduce also the system wide excitations corresponding to the experimentally observed DOTs. As we have a very limited number of parameters, we obtained state diagrams: in both models, DOTs appear when NC increases and $\lambda$ decreases (Figure 7D). In order to explore the possibility that some of the emergent dynamical properties of slices are due to their multilayer functional/anatomical organization, we constructed models of multilayer networks in which, in addition to intra-layer connections, every neuron $i$ has one connection to a neuron in the next layer (Figure 7E). Increasing the number of layers, the duration and Inter DOT Intervals of the simulated DOTs increased (Figure 7F).

We next compared the experimentally observed 
dynamical properties of native and dissociated networks with the corresponding simulated networks. The results are recapitulated in Figure $\mathbf{8}$ from the computation of optical traces $\Delta \mathrm{F} / \mathrm{F}_{\text {network }}$, and from the time series obtained with simulations. In both $\mathrm{HD}$ and MD cultures, $\Delta \mathrm{F} / \mathrm{F}_{\text {network }}$ has clear peaks but with a variable amplitude independently from the density of neurons (Figure 8A). A similar variability was obtained simulating a single layer network with Model 1 (Figure 8D). Experimentally, in the presence of Bicuculline, the peaks of $\Delta \mathrm{F} / \mathrm{F}_{\text {network }}$ have a more similar amplitude (Figure 8B), in line with the theoretical finding that decreasing the dissipation $\lambda$ enhances the probability of DOTs appearance (Figure 8E). In slices, $\Delta F / F_{\text {network }}$ has less frequent peaks with a uniform amplitude and longer duration comparing to dissociated cultures (Figure 8C) a pattern that is reproduced by our multilayer network as shown in Figure 8F. The DOTs experimentally observed in the cortex are longer than in the hippocampus (compare Figure 8G and $\mathbf{H}$ ) and have a specific time course during which the initial peak is followed by a longer plateau, with some oscillations not seen in dissociated cultures from the same tissue. Our multilayer models reproduce the occurrence of DOTs with a time course reminiscent of that seen in cortical slices (Figure 8I). Moreover, networks with an increased number of layers exhibit longer but less frequent DOTs. This is true even if they have the same number of neurons.

\section{Discussion}

Our results show that the spontaneous activity in cortical and hippocampal slices is dominated by the intermittency between a regime where optical signals are confined in several small regions and a regime where there is a global activation invading almost the entire visualized network, referred to as DOTs. This type of synchronized calcium transients were previously demonstrated to correspond to the depolarizing envelopes of spontaneous oscillations known as Up states, as shown by the correlation between individual neuronal spikes and network-wide bursting events (20). We therefore suggest that the DOTs observed in our experiments correspond to the spontaneous Up states that were previously described as events propagating throughout the cortex $(10,12)$.

DOTs appear as strong and fast increase of $\Delta \mathrm{F} / \mathrm{F}_{\text {network }}$, in a way reminiscent of spike generation in a single neuron - that is triggered by a positive feedback due to the voltage-gated conductance. Thus, at the network level, DOTs represent a positive feed-back which has been historically proposed to be a fundamental mechanism of information processing in the brain and to be at the basis of learning, memory and recognition $(43,44)$.

DOTs were observed in all examined neuronal networks, in those preserving the original cyto-architecture and after dissociation of the native tissue as well. Our results, however, demonstrate for the first time that the duration and intensity of DOTs depend on the original cyto-architecture of the cortex and of the hippocampus and on the overall balance between excitation and inhibition, as indicated by the action of APV (blocking NMDA glutamate receptors) and Bicuculine (blocking GABAergic receptors). In all types of networks analyzed, we found that the area of spontaneous events follows a power law distribution, in agreement with previous studies $(45,46)$. The power law behavior seen in the distribution of maximal areas of spontaneous events
A

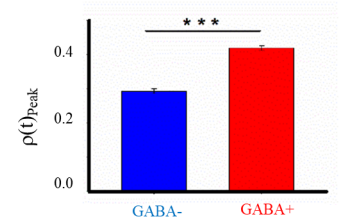

D

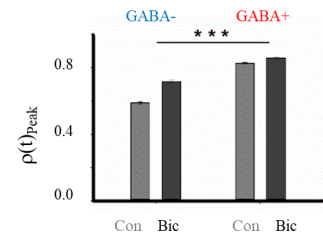

B

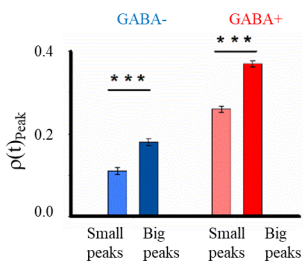

$\mathbf{E}$

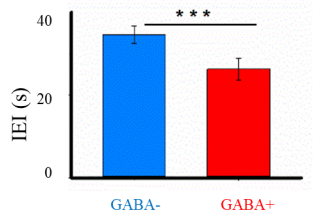

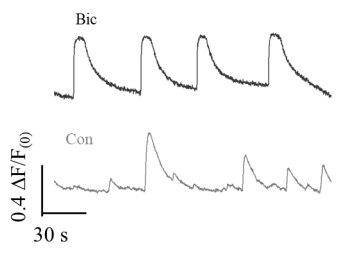

$\mathbf{F}$

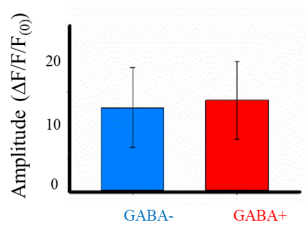

Fig. 6. Calcium transients from dissociated GABA+ and GABA- neurons. (A) Average $\rho(t))_{\text {Peak }}$, computed in $1 \mathrm{~s}$ time window, among pairs of GABA+ vs pairs of GABA- neurons in the network $(0.420 \pm 0.007$ vs $0.294 \pm 0.007$, respectively). (B) Same as (A) distinguishing between small and big peaks (defined by a threshold equal to the $30 \%$ of the maximal amplitude observed in each trace: $0.370 \pm 0.007$ vs $0.181 \pm 0.009$ for GABA+ neurons; $0.263 \pm 0.007$ vs $0.110 \pm 0.008$ for GABA- neurons). $n=605(3)$ pairs of neurons(sample) for $\mathrm{GABA}+, \mathrm{n}=870(3)$ pairs of neurons(sample) for GABA-. (C) Representative $\Delta F / F_{(0)}$ traces in control and after application of bicuculline $1 \mu \mathrm{M}$. (D) Average $\rho(\mathrm{t})_{\text {Peak }}$ in control and after bicuculline application, in GABA+ and GABA- neurons, computed in a time window of $10 \mathrm{~s}$ $(0.728 \pm 0.006$ and $0.865 \pm 0.003$ for GABA+ neurons in control and bicuculline conditions; $0.590 \pm 0.007$ and $0.828 \pm 0.005$ for GABAneurons in control and bicuculline conditions ). (E) Average IEI (s) between peaks of calcium transients in GABA+ and GABA- neurons $(26.07 \pm 2.13$ vs $34.62 \pm 2.62)$. $(F)$ Average amplitude $\left(\Delta F / F_{(0)}\right)$ in the two neuronal populations $(0.14 \pm 0.06$ vs $0.13 \pm 0.06)$. $n=73(3)$ neurons(sample) for GABA+, $n=105(3)$ neurons(sample) for GABA-). ${ }^{* \star} p<0.001$ One-Way ANOVA. 


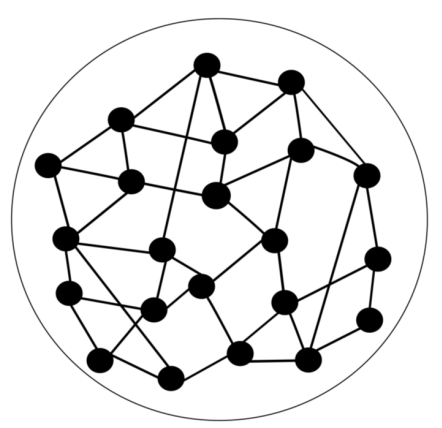

C

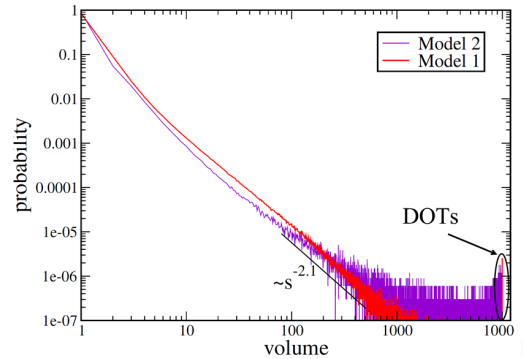

E

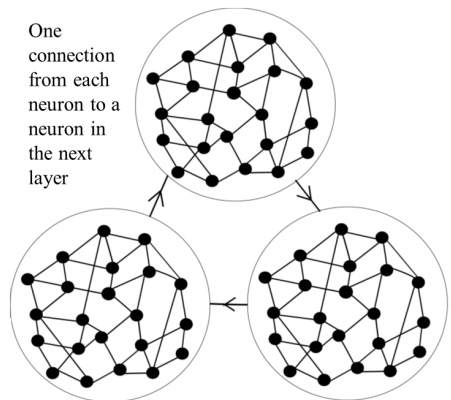

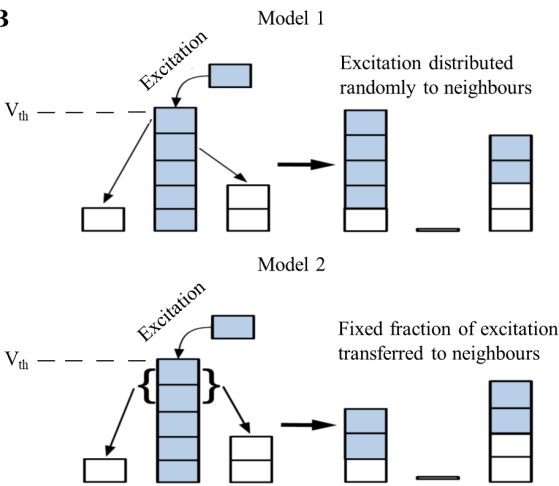

D
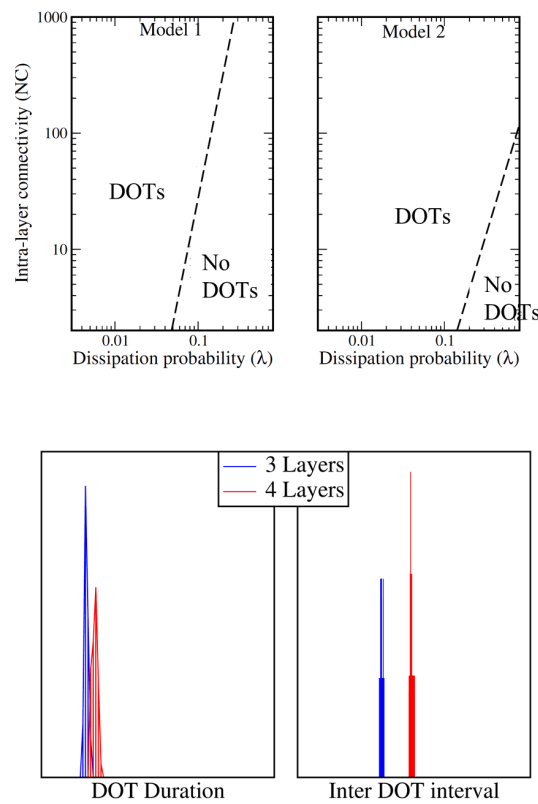

Fig. 7. Simulation setup and results. (A) Schematic diagram of the Single layer model of a network of N neurons with NC connections. (B) Schematic diagram of the mechanism of charge transfer in the two Models. (C) Distribution of avalanche volume for a network of $\mathrm{N}=$ 10,000 neurons in the two Models. (D) Phase diagram for the production of DOTs in terms of the dissipation and number of connections $\mathrm{NC}$, for the two Models. The dashed line is indicative of the regime where DOTs disappear as one moves along either of the axis. (E) Schematic diagram of the multi-layer model. Each layer consists of N neurons with NC connections as before. However, now every neuron has one additional projection to the neurons in the next layer. (F) Distribution of DOT duration (defined in units of number of simultaneous DOTs in successive layers, which maps to number of DOTs times $\Delta T$ ) and inter-DOT interval (defined in units of number of perturbations, i.e., simulation time steps) in a multilayer model with 3 layers (blue bars) as compared to one with 4 layers (red bars).

is in agreement with the predictions of the theory of Self Organized Criticality (47). This theory, however, assumes that the events under consideration are diluted in time and that only occasionally they occur simultaneously. Therefore, this central assumption of SOC does not hold for the optical transients observed in cortical and hippocampal slices, where we observed the simultaneous presence of tens of events at the same time.

In order to explain the experimental differences observed between the examined networks, we produced simplified models of neuronal networks with just $3 / 4$ parameters. The proposed modeling with simplified neural networks is based on the analysis of the interplay between different mechanisms at the basis of the dynamics of neural networks, such as the threshold $\mathrm{V}_{\text {th }}$ for spike initiation, the degree of connectivity $\mathrm{NC}$ and the dissipation $\lambda$. Neurons in cortical and hippocampal networks experience a large amount of synaptic inputs leading to a fluctuation of their membrane potential (48). Therefore, in order to take these mechanisms into account, Vth is not fixed and follows a power law distribution. Both fixed and power law distribution of $\mathrm{NC}$, instead, produce similar results in our model. However, experimental results support longtailed and lognormal distributions of NC and synaptic strength at the level of local circuits (see for review (49)). Our modeling introduces a rate of dissipation $\lambda$ of the charge accumulated in each neuron which has been inspired by statistical mechanics approaches $(47,50)$. This parameter is expected to describe in a lumped way some well-known mechanisms responsible for a time 
dependent decrease of the excitation, such as synaptic negative feedback, receptor desensitization and ionic channels inactivation. The few parameters used in our models suffice to reproduce the observed intermittency and collective excitation at the single layer level. In particular, collective (DOT - like) excitations take place when a neuron with a very high charge fires, distributing all of its charge to the neighbours and triggering a global avalanche. These neurons present thresholds in the tail of the power law distribution of $\mathrm{V}_{\mathrm{th}}$. Another important component required to observe DOT - like events is a sufficiently low value of dissipation $\lambda$. Finally, in order to obtain DOTs with broad peaks like those seen in cortical/ hippocampal slices, we need to introduce a multilayer architecture. The number of layers seems to determine the width and frequency of DOT - like events. In particular, networks with the highest number of layers exhibited longer but less frequent DOTs, in a way similar to what observed comparing cortical and hippocampal DOTs. This effect is not due to an increased number of total neurons in the network with more layers. We confirmed this result by simulating two networks with different numbers of layers, but the same number of neurons N. Multilayered networks might rather have a lower probability of relaxation - since two layers close to their critical state, i.e. the DOT, are more likely to be separated by bridging layers. In fact, we found that the network with more layers load a higher value of total charge before firing, not only creating broader but also less frequent DOTs.

Taken together, our findings provide new insights on the probability of neuronal avalanches that could be useful for the understanding of complex network disorders such as epilepsy. Large-scale models of hyperexcitable circuits, in fact, have been produced in order to predict the role of neuronal hubs in epileptic seizures (51). Our simplified models of networks represent an additional tool for simulating network disorders in brain regions with different functional and anatomical layers.

\section{Conclusion}

We assume that the experimentally observed differences in the frequency and duration of DOTs can be accounted for the presence of more anatomical/functional layers in cortical slices as compared to hippocampal slices, and in both type of slices as compared to dissociated cultures. We conclude that the network architecture and multilayer organization, together with the biophysical properties of neurons such as firing thresholds, connectivity, and degree of inhibition/desensitization, determine the emergent dynamical properties of the cortex and the hippocampus.
A

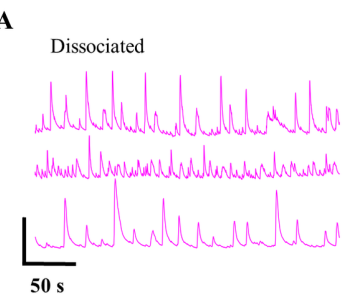

D

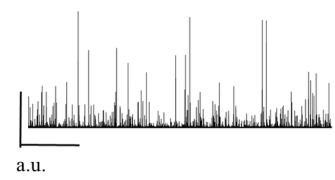

G

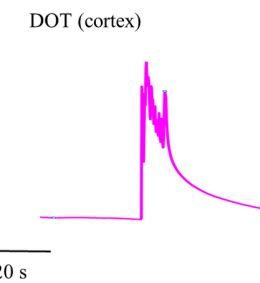

B

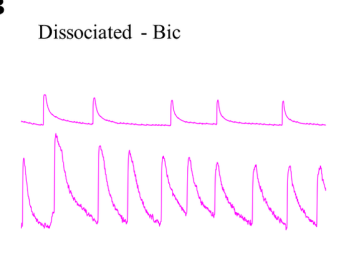

$\mathbf{E}$

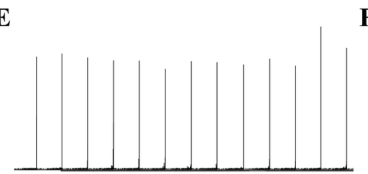

$\mathbf{H}$

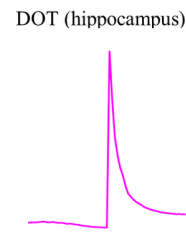

I
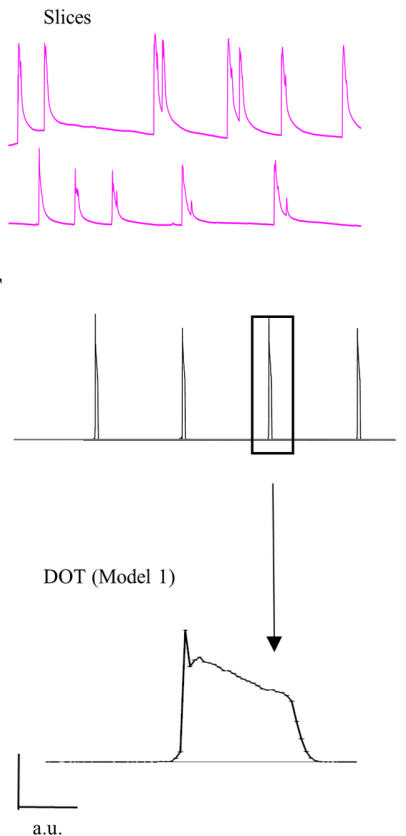

Fig. 8. Intermittency in dissociated and native networks and corresponding simulations. Experimental results are shown in purple, while time series obtained from the simulations are shown in black. $(A) \Delta F / F_{\text {network }}$ in dissociated MD/HD cultures. (B) $\Delta F / F_{\text {network }}$ in dissociated MD/HD cultures after application of Bicuculline $1 \mu \mathrm{M}$. (C) $\Delta F / F_{\text {network }}$ in some representative cortical slices. Scale bar, 0.1 $\Delta F / F_{\text {network. }}(D)$ Time series of a single-layer network from Model1. (E) Time series from the same simulated network after decreasing $\lambda$. (F) Time series from a multilayer network from Model1. In these plots, the x-axis are in units of number of perturbations, i.e., simulation time steps $\Delta \mathrm{T}$ (arbitrary units, a.u.). In the multilayer model, a $\Delta \mathrm{T}$ of 50,000 perturbation steps have been used. (G, H) Enlarged DOTs from $\Delta \mathrm{F} / \mathrm{F}_{\text {network }}$ of experimental recordings in cortical and hippocampal slices, respectively. Scale bar, $0.1 \Delta \mathrm{F} / \mathrm{F}_{\mathrm{n}}$ the simulation of the multilayer network. 


\section{Acknowledgements}

The authors would like to thank Michele Giugliano and Paolo Bonifazi for their useful comments on the early version of the manuscript.

\section{Conflict of interest}

Authors declare that there are no conflicts of interest.

\section{Funding body}

This work was supported by the 3315 project of Ningbo Institute of Materials Technology and Engineering, Chinese Academy of Sciences, Zhejiang province (China).

\section{Supplementary Information}

The supplemental material can be downloaded online at: https://stemedicine.org/index.php/stem/article/view/1

\section{References}

1. Faisal AA, Selen LPJ, Wolpert DM. Noise in the nervous system. Nat Rev Neurosci. 2008 Apr;9(4):292-303.

2. Luczak A, Barthó P, Marguet SL, Buzsáki G, Harris KD Sequential structure of neocortical spontaneous activity in vivo. Proc Natl Acad Sci USA. 2007 Jan 2;104(1):347-52.

3. Steriade M, Nuñez A, Amzica F. A novel slow (<1 Hz) oscillation of neocortical neurons in vivo: depolarizing and hyperpolarizing components. J Neurosci. 1993 Aug;13(8):3252-65.

4. Timofeev I, Grenier F, Steriade M. Disfacilitation and active inhibition in the neocortex during the naturalsleep-wake cycle: an intracellular study. Proc Natl Acad Sci USA. 2001 Feb 13;98(4):1924-9.

5. Petersen $\mathrm{CCH}$, Grinvald A, Sakmann B. Spatiotempora dynamics of sensory responses in layer $2 / 3$ of rat barre cortex measured in vivo by voltage-sensitive dye imaging combined with whole-cell voltage recordings and neuron reconstructions. J Neurosci. 2003 Feb 15;23(4):1298-309.

6. Sachidhanandam S, Sreenivasan V, Kyriakatos A, Kremer $\mathrm{Y}$, Petersen $\mathrm{CCH}$. Membrane potential correlates of sensory perception in mouse barrel cortex. Nat Neurosci. 2013 Nov;16(11):1671-7.

7. Vyazovskiy VV, Olcese U, Hanlon EC, Nir Y, Cirelli C, Tononi G. Local sleep in awake rats. Nature. 2011 Apr;472(7344):443-7.

8. Engel TA, Steinmetz NA, Gieselmann MA, Thiele A, Moore T, Boahen K. Selective modulation of cortical state during spatial attention. Science. 2016 02;354(6316):1140-4.

9. Timofeev I, Grenier F, Bazhenov M, Sejnowski TJ, Steriade M. Origin of slow cortical oscillations in deafferented cortica slabs. Cereb Cortex N Y N 1991. 2000 Dec;10(12):1185-99.

10. Sanchez-Vives MV, McCormick DA. Cellular and network mechanisms of rhythmic recurrent activity in neocortex. Nat Neurosci. 2000 Oct;3(10):1027-34.

11. Fanselow EE, Connors BW. The roles of somatostatinexpressing (GIN) and fast-spiking inhibitory interneurons in UP-DOWN states of mouse neocortex. J Neurophysiol. 2010Aug;104(2):596-606.

12. Compte A, Reig R, Descalzo VF, Harvey MA, Puccini GD, Sanchez-Vives MV. Spontaneous high-frequency $(10-80 \mathrm{~Hz})$ oscillations during up states in the cerebral cortex in vitro. $J$ Neurosci. 2008 Dec 17;28(51):13828-44.

13. Buzsáki G. Theta oscillations in the hippocampus. Neuron. 2002 Jan;33(3):325-40.

14. Sirota A, Buzsáki G. Interaction between neocortical and hippocampal networks via slow oscillations. Thalamus Relat Syst. 2005 Dec;3(04):245.

15. Markram H, Muller E, Ramaswamy S, Reimann MW Abdellah M, Sanchez CA, et al. Reconstruction and simulation of neocortical microcircuitry. Cell. 2015 Oct;163(2):456-92.

16. Scarpetta $\mathrm{S}$, de Candia A. Alternation of up and down states at a dynamical phase-transition of a neural network with spatiotemporal attractors. Front Syst Neurosci [Internet]. 2014 May 19;8:88.

17. Curto C, Sakata S, Marguet S, Itskov V, Harris KD. A simple model of cortical dynamics explains variability and state dependence of sensory responses in urethane-anesthetized auditory cortex. J Neurosci. 2009 Aug 26;29(34):10600-12.

18. Goodfellow M, Glendinning P. Mechanisms of intermittent state transitions in a coupled heterogeneous oscillator model of epilepsy. J Math Neurosci. 2013 Aug 14;3:17.

19. Tamamaki N, Yanagawa Y, Tomioka R, Miyazaki J-I, Obata K, Kaneko T. Green fluorescent protein expression and colocalization with calretinin, parvalbumin, and somatostatin in the GAD67-GFP knock-in mouse. J Comp Neurol. 2003 Dec 1;467(1):60-79.

20. Cossart R, Ikegaya Y, Yuste R. Calcium imaging of cortical networks dynamics. Cell Calcium. 2005 May;37(5):451-7.

21. Yang W, Yuste R. In vivo imaging of neural activity. Nat Methods. 2017 Mar 31;14(4):349-59.

22. Gähwiler BH, Capogna M, Debanne D, McKinney RA Thompson SM. Organotypic slice cultures: a technique has come of age. Trends Neurosci. 1997 Oct;20(10):471-7.

23. Stoppini L, Buchs PA, Muller D. A simple method for organotypic cultures of nervous tissue. J Neurosci Methods. 1991 Apr;37(2):173-82.

24. Clauset A, Shalizi C, Newman M. Power-Law Distributions in Empirical Data. SIAM Rev. 2009 Nov 4;51(4):661-703.

25. Regad T, Bellodi C, Nicotera P, Salomoni P. The tumor suppressor $\mathrm{Pml}$ regulates cell fate in the developing neocortex. Nat Neurosci. 2009 Feb;12(2):132-40.

26. Douglas RJ, Martin KAC. Neuronal circuits of the neocortex. Annu Rev Neurosci. 2004;27:419-51.

27. Narahashi T, Moore JW, Scott WR. Tetrodotoxin Blockage of Sodium Conductance Increase in Lobster Giant Axons. J Gen Physiol. 1964 May 1;47(5):965-74.

28. Gilbride CJ. The hyperexcitability of dentate granule neurons in organotypic hippocampal slice cultures is due to reorganization of synaptic inputs in vitro. Physiol Rep. 2016 Oct 1;4(19):n/a-n/a.

29. Tamura A, Yamada N, Yaguchi Y, Machida Y, Mori I, Osanai $M$. Both neurons and astrocytes exhibited tetrodotoxinresistant metabotropic glutamate receptor-dependent spontaneous slow $\mathrm{Ca} 2+$ oscillations in striatum. PLOS ONE. 2014 Jan 15;9(1):e85351.

30. Mazzoni A, Broccard FD, Garcia-Perez E, Bonifazi P, Ruaro $\mathrm{ME}$, Torre $\mathrm{V}$. On the dynamics of the spontaneous activity in neuronal networks. PLoS ONE. 2007 May 9;2(5):e439.

31. Cossart R, Aronov D, Yuste R. Attractor dynamics of network UP states in the neocortex. Nature. 2003 May 15;423(6937):283-8.

32. Mao B-Q, Hamzei-Sichani F, Aronov D, Froemke RC, Yuste R. Dynamics of spontaneous activity in neocortical slices. Neuron. 2001 Dec;32(5):883-98.

33. Piet R, Jahr CE. Glutamatergic and purinergic receptormediated calcium transients in Bergmann glial cells. J Neurosci. 2007 Apr 11;27(15):4027-35.

34. Rojas A, Wetherington J, Shaw R, Serrano G, Swanger S, Dingledine R. Activation of group I metabotropic glutamate receptors potentiates heteromeric kainate receptors. Mol Pharmacol. 2013 Jan;83(1):106-21.

35. Sengupta M, Thirumalai V. AMPA receptor mediated synaptic excitation drives state-dependent bursting in Purkinje neurons of zebrafish larvae. eLife. 2015 Sep 29;4:e09158.

36. Parga N, Abbott LF. Network model of spontaneous activity exhibiting synchronous transitions between up and down states. Front Neurosci. 2007 Oct 15;1(1):57-66.

37. Wadsworth GP, Bryan JG. Introduction to probability and random variables. McGraw-Hill; 1960. 312 p.

38. Charles AC, Merrill JE, Dirksen ER, Sanderson MJ. Intercellular signaling in glial cells: calcium waves and oscillations in response to mechanical stimulation and glutamate. Neuron. 1991 Jun;6(6):983-92. 
39. Ito D, Tamate H, Nagayama M, Uchida T, Kudoh SN, Gohara K. Minimum neuron density for synchronized bursts in a rat cortical culture on multi-electrode arrays. Neuroscience. 2010 Nov 24;171(1):50-61.

40. Pozzi D, Ban J, Iseppon F, Torre V. An improved method for growing neurons: Comparison with standard protocols. J Neurosci Methods. 2017 Mar;280:1-10.

41. Ulloa Severino FP, Ban J, Song Q, Tang M, Bianconi G, Cheng G, et al. The role of dimensionality in neuronal network dynamics. Sci Rep. 2016 Jul 11;6:29640.

42. Gentet LJ, Avermann M, Matyas F, Staiger JF, Petersen $\mathrm{CCH}$. Membrane potential dynamics of GABAergic neurons in the barrel cortex of behaving mice. Neuron. 2010 Feb;65(3):422-35

43. Fukushima K. A hierarchical neural network model for associative memory. Biol Cybern. 1984 Apr 1;50(2):105-13.

44. Rolls ET. Cerebral cortex: principles of operation. First edition. Oxford New York, NY: Oxford University Press; 2016. $958 p$

45. Beggs JM, Plenz D. Neuronal avalanches in neocortical circuits. J Neurosci. 2003 Dec 3;23(35):11167-77.

46. Klaus A, Yu S, Plenz D. Statistical analyses support power law distributions found in neuronal avalanches. PLoS ONE. 2011 May 26;6(5):e19779.

47. Bak P, Tang C, Wiesenfeld K. Self-organized criticality: an explanation of the 1/f noise. Phys Rev Lett. 1987 Jul 27;59(4):381-4.

48. $\mathrm{Hu}$ W, Bean BP. Differential control of axonal and somatic resting potential by voltage-dependent conductances in cortical layer 5 pyramidal neurons. Neuron. 2018 Mar 21;97(6):13151326.e3.

49. Schröter M, Paulsen O, Bullmore ET. Micro-connectomics: probing the organization of neuronal networks at the cellular scale. Nat Rev Neurosci. 2017;18(3):131-46.

50. Marsili M, Valleriani A. Self Organization of Interacting Polya Urns. Eur Phys J B. 1998 Jul;3(4):417-20.

51. Morgan RJ, Soltesz I. Nonrandom connectivity of the epileptic dentate gyrus predicts a major role for neuronal hubs in seizures. Proc Natl Acad Sci USA. 2008 Apr 22;105(16):617984. 